\title{
Mapeamento Sistemático de Artigos do Estado da Arte sobre Experimentos com Pensamento Computacional no Ensino Básico
}

\author{
Luís Antônio dos Santos Silva ${ }^{1}$, Maria Augusta Silveira Netto Nunes ${ }^{12}$ \\ ${ }^{1}$ Programa de Pós-Graduação em Ciência da Computação \\ Universidade Federal de Sergipe (UFS) - São Cristovão - Sergipe - Brasil \\ ${ }^{2}$ Programa de Pós-Graduação em Informática (PPGI) \\ Universidade Federal do Estado do Rio de Janeiro (UNIRIO) - \\ Rio de Janeiro - Rio de Janeiro - Brasil \\ antonio.silva@dcomp.ufs.br, gutanunes@gmail.com
}

\begin{abstract}
Computational Thinking is an important skill for the 21st century, so it can be of immense help in meeting SBC's Challenge to bring universal and participatory knowledge to every Brazilian citizen. The aim of this article is to carry out a Systematic Literature Mapping (SLM) of the state of the art articles on experiments with Computational Thinking in Basic Education. 430 articles were selected after inclusion and exclusion criteria, from 5494 articles obtained. The results show a variety of disciplines in which the studies are carried out, as well as several materials, tests used and few articles outside the axis of Science, Technology, Engineering and Mathematics (STEM).
\end{abstract}

Resumo. O Pensamento Computacional é uma importante habilidade para o século XXI, por isso pode ser de imensa ajuda para o cumprimento do Desafio da SBC de levar conhecimento universal e participativo a todo cidadão brasileiro. O objetivo deste artigo é realizar um Mapeamento Sistemático da Literatura (MSL) de artigos do estado da arte sobre experimentos com Pensamento Computacional no Ensino Básico. Foram selecionados 430 artigos depois do critérios de inclusão e exclusão, de 5494 artigos obtidos. Os resultados mostram uma variedade de disciplinas em que os estudos são realizados, assim como vários materiais, testes utilizados e poucos artigos fora do eixo da Ciência, Tecnologia, Engenharia e Matemática (STEM).

\section{Introdução}

O termo Pensamento Computacional surgiu e foi descrito por [Papert et al. 1971] em seu artigo Twenty things to do with a computer, porém desde sua publicação o Pensamento Computacional (PC) era pouco difundido e não existia um movimento para sua propagação [Brackmann 2017], até que [Wing 2006] publicou o artigo Computational Thinking, no qual a autora alega que conceitos da Ciência da Computação (CC), podem ser agregados a outras matérias do currículo escolar.

O PC pode ser definido como "processos de pensamento envolvidos na formulação de problemas e suas soluções, sendo que as soluções devem ser representadas de forma que possa ser realizada por agentes de processamento de informações" [Cuny et al. 2010]. 
Outra definição é dada por [Romero et al. 2019], na qual destacam o PC como um conjunto de estratégias cognitivas e metacognitivas relacionadas à modelagem de conhecimento e de processos, à abstração, ao algoritmo, à identificação, à decomposição e à organização de estruturas complexas e conjuntos lógicos.

Segundo a [OECD 2020] há uma defasagem no currículo escolar nos sistemas educacionais. Dentro dessa situação, no Brasil foi elaborada a Base Nacional Comum Curricular (BNCC), a qual adota padrões internacionais por meio de competências gerais e específicas que precisam ser desenvolvidas[MEC 2019], sendo o PC citado pela BNCC como uma habilidade que estudantes precisam ser estimulados a desenvolver [MEC 2019. p.528], havendo iniciativas nessa área que podem virar politicas públicas [CIEB 2016] e em sua formulação são apoiadas pelo Centro de Inovação para a Educação Brasileira (CIEB).

Por isso, o PC é alvo de várias pesquisas brasileiras [de Oliveira et al. 2014, Raabe et al. 2017. França and Tedesco 2017, de França and Tedesco 2015] e estrangeiras [Relkin et al. 2021, Rich et al. 2021, Kale and Yuan 2021]. Sendo considerado por [Romero 2016, Romero et al. 2019] uma competência do século XXI que os estudantes precisam desenvolver, o qual está em conformidade com a BNCC [MEC 2019, p.528], com a função da Sociedade Brasileira de Computação (SBC) [SBC 2018] e com a missão da Comissão Especial de Informática na Educação (CEIE) [CEIE 2018]. Essas entidades apoiam o ensino de computação no Brasil e que tem como Desafio, segundo o relatório da SBC o acesso participativo e universal do cidadão brasileiro ao conhecimento [SBC 2015], sendo o Brasil um país onde grande parte das escolas não tem infraestrutura adequada [Brasil 2017] . Contudo, é preciso também medir o sucesso desses experimentos para que se saiba o quantitativo de desenvolvimento do PC antes e depois deles, afinal aquilo que não se pode medir, também não se pode melhorar [de Freitas et al. 2017].

Além disso, o PC tem um importante papel nos estudos que utilizam disciplinas diretamente ligadas a área de Science, Technology, Engineering and Mathematics (STEM) no ensino básico[Mecca et al. 2021, Rowe et al. 2021], mas que também poderia ser usado em outras áreas do conhecimento.

Este artigo tem como objetivo realizar um Mapeamento Sistemático da Literatura (MSL) dos artigos do estado da arte sobre experimentos com Pensamento Computacional no Ensino Básico. Isso é feito por meio da Questão Primária de Pesquisa (QPP) apresentada na Seção 2.

O restante do artigo está organizado como segue: na Seção 2 apresenta-se a metodologia aplicada; na Seção 3 apresenta-se a análise dos resultados obtidos no mapeamento; na Seção 4 apresenta-se as ameaças à validade; na Seção 5 apresenta-se as conclusões do artigo; e por fim, as referências.

\section{Metodologia}

Neste artigo utiliza-se o método de Mapeamento Sistemático da Literatura (MSL) como apresentado em [Silva et al. 2018], proposto originalmente por [Petersen et al. 2008]. Este método é caracterizado por etapas pré-definidas, tais como: (1) definição das questões de pesquisa; (2) identificação dos estudos por meio da escolha das palavras-chave e montagem da string de busca; (3) seleção de critérios de inclusão e exclusão dos estudos primários, selecionando os estudos relevantes; seguido pela avaliação da qualidade 
dos estudos; (4) sintetizando e analisando-os por meio da discussão dos resultados. Na realização desse MSL foi usada a Plataforma Evidências - Módulo SUMARIZY $\llbracket$ ferramenta projetada para pesquisadores para realizarem revisões sistemáticas da literatura e meta-análises criada pelo CIEB e que ajudou na condução deste MSL.

\subsection{Questões de Pesquisa}

Este mapeamento buscou analisar a seguinte QPP: "Qual o panorama atual de pesquisa dos experimentos sobre o Pensamento Computacional no Ensino Básico?". Com o objetivo de buscar dados mais aprofundados, foram acrescidas as Questões de Pesquisa Secundárias (QPS). As questões de pesquisa secundárias bem como os dados a serem extraídos propostos para esse mapeamento estão dispostos e selecionados como apresentados na Tabela 1.

Tabela 1 - Questōes de pesquisa e dados a serem extraídos

\begin{tabular}{|c|c|}
\hline \multicolumn{2}{|c|}{ Questão de Pesquisa Primária } \\
\hline $\begin{array}{r}\text { Qual o panorama atual de pesquisa dos exp } \\
\text { no Ensi }\end{array}$ & $\begin{array}{l}\text { imentos sobre o Pensamento Computacional } \\
\text { Básico? }\end{array}$ \\
\hline Questôes de Pesquisa Secundárias & Dados a serem extraídos \\
\hline $\begin{array}{l}\text { Quais disciplinas são alvo da intervenção } \\
\text { realizada? }\end{array}$ & Português, Matemática, Geografia, etc. \\
\hline $\begin{array}{c}\text { Quais materiais são utilizados para o } \\
\text { experimento? }\end{array}$ & Robôs, Atividades desplugadas, etc. \\
\hline $\begin{array}{l}\text { O experimento está fora do eixo Science, } \\
\text { Technology, Engineering e Mathematics } \\
\text { (STEM)? }\end{array}$ & Sim, não \\
\hline $\begin{array}{l}\text { Qual teste é feito para medir o Pensamento } \\
\text { Computacional? }\end{array}$ & $\begin{array}{c}\text { Testes feitos pelos próprios autores, Tarefas } \\
\text { Brebas, etc. }\end{array}$ \\
\hline
\end{tabular}

\subsection{Identificação dos Estudos}

O mapeamento foi conduzido primeiramente nas bases de dados nacionais como SciELO $^{2}$, Educapes $?^{3}$ e Educ @ $4^{4}$, além da base de publicações indexadas pela Comissão Especial de Informática na Educação ${ }^{5}$ (CEIE), a saber: Revista Brasileira de Informática na Educação (RBIE), Simpósio Brasileiro de Informática na Educação (SBIE), Anais do Workshop de Informática na Escola (WIE), Anais dos Workshops do Congresso Brasileiro de Informática na Educação (WCBIE), Jornada de Atualização em Informática na Educação, Anais do Workshop de Desafios da Computação Aplicada à Educação e SBC OpenLib ${ }^{6}$ (SOL). Além disso, incluímos a base de estudos da revista Renote ${ }^{7}$. A pesquisa também foi estendida a bases de dados estrangeiras como ACM Digital Library ${ }^{8} \mathrm{e}$ IEEE Xplore Digital Library $y^{9}$, além das bases ISI Web of Science ${ }^{10}$, Science@Direct ${ }^{11}$. Scopus ${ }^{12}$ e Springer Link ${ }^{13}$

\footnotetext{
${ }^{1}$ https://qeed.nees.com.br/

${ }^{2}$ https://www.scielo.org/

${ }^{3}$ https://educapes.capes.gov.br/

${ }^{4}$ https://www.fcc.org.br/fcc/educ

${ }^{5}$ https://www.br-ie.org/pub/index.php/index

${ }^{6} \mathrm{https}: / /$ sol.sbc.org.br/index.php/indice

${ }^{7}$ https://seer.ufrgs.br/renote

${ }^{8}$ https://dl.acm.org/

${ }^{9} \mathrm{https} / / /$ ieeexplore.ieee.org/Xplore/home.jsp

${ }^{10} \mathrm{https} / / /$ login.webofknowledge.com/

${ }^{11} \mathrm{https}: / / \mathrm{www}$. sciencedirect.com/

${ }^{12} \mathrm{https}: / / \mathrm{www}$. scopus.com/

${ }^{13}$ https://link.springer.com/
} 


\subsubsection{Termos utilizados na busca}

Presente na ferramenta utilizada (SUMARIZE- CIEB), a definição dos termos de pesquisa seguiram a estratégia PICOC (População, Intervenção, Comparação, Resultado/Outcome e Contexto) [BA and Charters 2007], orientando a seleção dos estudos primários direcionando o escopo do artigo e que foi definido neste mapeamento como População (crianças, adolescentes, jovem, pré-adolescente), Intervenção (experimento do Pensamento Computacional), Comparação (antes e após a intervenção), Resultado (desenvolvimento do Pensamento Computacional) e Contexto (escola, educação básica, aprendizagem, ensino, aula). Para a busca, foram considerados os estudos e soluções que possuíssem os termos listados na Tabela 2 e seus respectivos sinônimos em seus títulos, resumos ou palavras-chaves. Para as bases estrangeiras foram utilizadas as respectivas traduções.

Tabela 2 - Palavras-chave, sinônimos e termos em inglês utilizados nas buscas

\begin{tabular}{|c|c|c|}
\hline $\begin{array}{c}\text { Palavras- } \\
\text { chave }\end{array}$ & Sinônimos & $\begin{array}{c}\text { Termos correspondente em } \\
\text { Inglês }\end{array}$ \\
\hline $\begin{array}{c}\text { pensamento } \\
\text { computacion } \\
\text { al }\end{array}$ & $\begin{array}{c}\text { pensamento algorítmico, } \\
\text { pensamento de programação, } \\
\text { pensamento programático }\end{array}$ & $\begin{array}{c}\text { computational thinking, } \\
\text { algorithmic thinking, } \\
\text { programming thinking }\end{array}$ \\
\hline educação & $\begin{array}{c}\text { educação infantil, criança, } \\
\text { ensino fundamental, educaçäo } \\
\text { secundária, educação } \\
\text { primária, jovem, pré-escola, } \\
\text { ensino médio, jardim de } \\
\text { infância }\end{array}$ & $\begin{array}{c}\text { educa*, early childhood } \\
\text { education, children, middle } \\
\text { school, secondary education, } \\
\text { primary education, young } \\
\text { people, preschool, elementary } \\
\text { school, junior high, } \\
\text { kindergarten, pre- k }\end{array}$ \\
\hline
\end{tabular}

As buscas foram construídas com operadores booleanos OR e AND entre os termos buscados e seus sinônimos. Também foi utilizado o operador coringa $\left({ }^{*}\right)$ nas palavras com variações nas bases que davam suporte a esse operador, gerando assim a string de busca da Tabela 3. As buscas foram feitas por publicações recentes sobre o tema, contemplando os anos 2019, 2020 e até maio de 2021.

Tabela 3 - Strings de busca

\begin{tabular}{|c|c|}
\hline Português & Inglês \\
\hline (("pensamento computacional" OR & (("Computational thinking" OR \\
"pensamento algorítmico" OR & "Algorithmic thinking" OR \\
"pensamento de programação" OR & "Programming thinking") AND \\
"pensamento programático") AND & ("education" OR "early childhood \\
("educação" OR "educação infantil" OR & education" OR "Children" OR "Middle \\
"criança" OR "ensino médio" OR & School" OR "Secondary Education" \\
"educação secundária" OR "Educação & OR "Primary Education" OR "Young \\
primária" OR "jovens" OR "pré-escola" & people" OR "Preschool" OR \\
OR "ensino médio" OR "Jardim de & "Elementary School" OR "Junior high" \\
infância")) & OR "Kindergarten" OR "pre- k")) \\
\hline
\end{tabular}

\subsection{Critérios de Seleção}

As pesquisas selecionadas passaram por um primeiro filtro, aplicando os critérios de inclusão (CI) e exclusão (CE) sendo eles: (CI1) Acessível via web de maneira gratuita, (CI2) Artigos com idioma em inglês e português, (CI3) Estudos publicados a partir de 2019, (CI4) Somente estudos primários, (CI5) Estudos realizados na Educação 
Básica, (CI6) Somente estudos com intervenções caso-controle (experimental), quaseexperimental ou longitudinal, (CI7) Estudos que tenham como fim o desenvolvimento do Pensamento Computacional; e exclusão (CE), mostrados a seguir: (CE1) Estudos Duplicados, (CE2) Falta de disponibilidade para download de maneira gratuita, (CE3) Estudos publicados como resumos ou resumo expandido ou prefácio de periódicos e eventos,(CE4) Estudos que não contemplam a temática, (CE5) Estudos não realizados na Educação Básica e (CE6) Estudos publicados com ano inferior a 2019.

No segundo filtro, as pesquisas foram avaliadas por questões de qualidade. Para que fossem respondidas foi necessária a leitura completa da publicação/artigo. As Questões de Qualidade (QQ) estão detalhadas na Tabela 4.

Tabela 4 - Questōes de Qualidade

\begin{tabular}{|c|c|}
\hline \multicolumn{2}{|c|}{ Questões de Qualidade (QQ) } \\
\hline $\begin{array}{l}\text { O estudo atribui o desenvolvimento do } \\
\text { Pensamento Computacional como um dos } \\
\text { objetivos do experimento? }\end{array}$ & Sim (2.0); Não (0.0) \\
\hline $\begin{array}{l}\text { A intervenção realizada utiliza testes para } \\
\text { avaliar o desenvolvimento do Pensamento } \\
\text { Computacional? }\end{array}$ & Sim (2.0); Não (0.0) \\
\hline $\begin{array}{l}\text { São mencionadas as ameaças à validade e } \\
\text { também como essas ameaças afetam os } \\
\text { resultados e conclusões? }\end{array}$ & Sim (2.0); Não (0.0) \\
\hline $\begin{array}{l}\text { O artigo descreve de forma clara o material } \\
\text { utilizado? }\end{array}$ & Sim (2.0); Não (0.0) \\
\hline $\begin{array}{l}\text { Há interdisciplinaridade na intervenção } \\
\text { realizada? }\end{array}$ & Sim (2.0); Não (0.0) \\
\hline
\end{tabular}

A Pontuação de Avaliação de Qualidade é resultado da soma da pontuação de cada pergunta, sendo a Pontuação Máxima $(10,0)$ que o estudo pode alcançar após essa soma e uma Pontuação de Corte $(8,0)$ na qual a publicação que ficou com pontuação menor ou igual a ela foi rejeitada. Assim reduzindo para um total de 28 estudos apresentados na Tabela 5. 
Tabela 5 - Detalhamento do Processo de Seleção dos estudos

\begin{tabular}{|c|c|c|c|}
\hline Bases & $\begin{array}{c}\text { Estudos } \\
\text { Primários }\end{array}$ & $\begin{array}{c}\text { Estudos Relevantes } \\
\text { Selecionados após } \\
\text { Cl e CE }\end{array}$ & $\begin{array}{c}\text { Estudos Relevantes } \\
\text { após Questões de } \\
\text { Qualidade }\end{array}$ \\
\hline Scielo & 281 & 6 & 0 \\
\hline CEIE & 83 & 10 & 6 \\
\hline Renote & 3 & 0 & 0 \\
\hline EDUC@ & 43 & 0 & 0 \\
\hline Educapes & 162 & 9 & 0 \\
\hline Scopus & 185 & 0 & 9 \\
\hline ACM & 230 & 15 & 3 \\
\hline IEEE & 245 & 23 & 0 \\
\hline $\begin{array}{c}\text { Science } \\
\text { Direct }\end{array}$ & 3186 & 80 & 2 \\
\hline Springer Link & 405 & 28 & 3 \\
\hline PEPSIC & 124 & 5 & 5 \\
\hline $\begin{array}{c}\text { Web of } \\
\text { Science }\end{array}$ & 75 & 5 & 28 \\
\hline Total & 5494 & 430 & \\
\hline
\end{tabular}

\section{Análise dos Resultados}

Os 28 estudos relevantes estão organizados nesse link ${ }^{14}$, onde é possível visualizar a referência completa do estudo e um código de identificação para os Estudos do Pensamento Computacional (EPC), acrescido de um valor numérico, que será utilizado como referência. A seguir, primeiramente são apresentadas as análises para responder às questões de pesquisa secundárias e, por último, a análise da questão primária de pesquisa. Nas Tabelas os estudos estão divididos entre estudos brasileiros (estudos em que o local de coleta de dados foi no Brasil) e estudos estrangeiros (estudos em que o local coleta de dados não foi no Brasil).

QPS1 - Quais disciplinas são alvo do experimento realizado?

Quanto à disciplina em que foi realizado o estudo, a maior parte dos experimentos não focou em nenhuma disciplina específica (10), logo seguido por Matemática (4), Programação (4), Ciências (3), Robótica (2), Música (2) e também aparecem outras disciplinas em menor quantidade, conforme a Tabela 6.

$\sqrt[14]{\text { https://github.com/luigi20/Estudos-sobre-Experimentos-com-PC }}$ 
Tabela 6 - Disciplinas nas quais os experimentos são aplicados em contexto brasileiro e estrangeiro

\begin{tabular}{|c|c|c|c|}
\hline Disciplinas & Estudos Brasileiros & Estudos Estrangeiros & $\begin{array}{c}\text { Quantitativo de } \\
\text { Estudos }\end{array}$ \\
\hline Nenhuma & $\begin{array}{c}\text { EPC15, EPC26, } \\
\text { EPC27, EPC28 }\end{array}$ & $\begin{array}{c}\text { EPC04, EPC18, EPC19, } \\
\text { EPC20, EPC23, EPC25 }\end{array}$ & 10 \\
\hline Matemática & EPC01 & EPC06, EPC16, EPC22 & 4 \\
\hline Programação & - & EPC08, EPC11, EPC12, & 4 \\
\hline EPC13 & EPC03, EPC10, EPC17 & 3 \\
\hline Música & - & EPC08 & 2 \\
\hline Robótica & EPC02 & EPC09, EPC24 & 2 \\
\hline Física & - & EPC05 & 1 \\
\hline Informática & - & EPC14 & 1 \\
\hline Tecnologia da \\
Informação
\end{tabular}

QPS2 - Quais materiais são utilizados para o experimento?

Quanto aos materiais utilizados nos experimentos: Atividades Desplugadas (9), Scratch (8) e Robôs/Kit de Robótica (6) correspondem aos materiais mais utilizados, seguidos de outros em menor número na Tabela 7.

Tabela 7 - Materiais utilizados nos experimentos em contexto brasileiro e em contexto estrangeiro

\begin{tabular}{|c|c|c|c|}
\hline Materiais & Estudos Brasileiros & Estudos Estrangeiros & $\begin{array}{c}\text { Quantitativo } \\
\text { de Estudos }\end{array}$ \\
\hline $\begin{array}{c}\text { Atividades } \\
\text { Desplugadas }\end{array}$ & $\begin{array}{c}\text { EPC01, EPC02, } \\
\text { EPC15, EPC27, } \\
\text { EPC28 }\end{array}$ & $\begin{array}{c}\text { EPC12, EPC19, EPC23, } \\
\text { EPC24 }\end{array}$ & 9 \\
\hline Scratch & EPC26 & $\begin{array}{c}\text { EPC04, EPC06, EPC07, } \\
\text { EPC11, EPC18, EPC19, } \\
\text { EPC20 }\end{array}$ & 8 \\
\hline $\begin{array}{c}\text { Robôs/Kit de } \\
\text { Robótica }\end{array}$ & - & $\begin{array}{c}\text { EPC03, EPC06, EPC09, } \\
\text { EPC10, EPC22, EPC25 }\end{array}$ & 6 \\
\hline Planilhas Eletrônicas & - & EPC13, EPC14 & 2 \\
\hline $\begin{array}{c}\text { Linguagem de } \\
\text { Programação }\end{array}$ & - & EPC17, EPC22 & 2 \\
\hline Hora do Código & EPC26 & - & 1 \\
\hline ScratchX & - & EPC25 & 1 \\
\hline HQ's & EPC01 & EPC08 & 1 \\
\hline Material Reciclado & - & EPC09 & 1 \\
\hline $\begin{array}{c}\text { Sistema Físico de } \\
\text { Cartões }\end{array}$ & - & EPC10 & 1 \\
\hline $\begin{array}{c}\text { Programação de } \\
\text { Blocos Visuais }\end{array}$ & - & EPC16 & 1 \\
\hline $\begin{array}{c}\text { Ambiente de } \\
\text { programação }\end{array}$ & - & EPC25 & 1 \\
\hline App Inventor & - & & \\
\hline
\end{tabular}

QPS3 - O experimento está fora do eixo Science, Technology, Engineering e Mathematics (STEM)?

De acordo com os estudos selecionados, apenas 3 artigos, sendo 2 estudos brasileiros (EPC01, EPC02) e um estrangeiro (EPC08) realizam experimentos fora do eixo STEM, mostrado na Tabela 8. 
Tabela 8 - Estudos selecionados dentro e fora do eixo STEM e por estudos brasileiros e estrangeiros

\begin{tabular}{|c|c|c|c|}
\hline Eixo STEM & Estudos Brasileiros & Estudos Estrangeiros & $\begin{array}{c}\text { Quantitativo de } \\
\text { Estudos }\end{array}$ \\
\hline Sim & EPC15, EPC26, & EPC03, EPC04, & 25 \\
& EPC27, EPC28 & EPC05, EPC06, & \\
& & EPC07, EPC09, & \\
& & EPC10, EPC11, & \\
& & EPC12, EPC13, & \\
& & EPC14, EPC16, & \\
& & EPC17, EPC18, & \\
& & EPC19, EPC20, & \\
& & EPC21, EPC22, & \\
& & EPC25 & \\
\hline Não & EPC01, EPC02 & EPC08 & 3 \\
\hline
\end{tabular}

QPS4 - Qual o teste feito para avaliar o Pensamento Computacional?

Conforme a amostra apresentada na Tabela 9, testes criados pelos próprios autores (7) e tarefas Brebas 45 (5) são os que mais aparecem, seguidos Computational Thinking Test (1) ou adaptações (2) ou traduções (1) do mesmo, assim como outros testes em menor número.

Tabela 9 - Distribuição dos Estudos Selecionados pelos Teste do Pensamento Computacional Aplicados e por Estudos Brasileiros e Estrangeiros

\begin{tabular}{|c|c|c|c|}
\hline Testes & $\begin{array}{l}\text { Estudos } \\
\text { Brasileiros }\end{array}$ & $\begin{array}{c}\text { Estudos } \\
\text { Estrangeiros }\end{array}$ & $\begin{array}{l}\text { Quantitativo de } \\
\text { Estudos }\end{array}$ \\
\hline $\begin{array}{c}\text { Testes criados pelos próprios } \\
\text { autores }\end{array}$ & EPC28 & $\begin{array}{l}\text { EPC03, EPC13, } \\
\text { EPC14, EPC17, } \\
\text { EPC18, EPC20 }\end{array}$ & 7 \\
\hline Tarefas Brebas & - & $\begin{array}{l}\mathrm{EPC} 05, \mathrm{EPC} 19, \\
\mathrm{EPC} 22, \mathrm{EPC} 23, \\
\mathrm{EPC} 24\end{array}$ & 5 \\
\hline $\begin{array}{l}\text { Computational Thinking Test } \\
\text { [Román-Gonzalez et al. 2017] } \\
\text { traduzido por [Brackmann 2017] }\end{array}$ & $\begin{array}{c}\text { EPC02, EPC15, } \\
\text { EPC27 }\end{array}$ & - & 3 \\
\hline $\begin{array}{c}\text { Adaptação do Computational } \\
\text { Thinking Test [Román-Gonzalez et } \\
\text { al. 2017] }\end{array}$ & EPC26 & EPC09 & 2 \\
\hline $\begin{array}{l}\text { Problem Solving Inventory for } \\
\text { Children at Elementary Level } \\
\text { [Serin et al. 2010] }\end{array}$ & - & EPC04, EPC21 & 2 \\
\hline $\begin{array}{c}\text { Aplicação de Exercícios adaptados } \\
\text { de [Yagci 2019] }\end{array}$ & EPC01 & - & 1 \\
\hline $\begin{array}{c}\text { Computational Thinking Skill } \\
\text { Levels Scale [Korkmaz et al. 2017] }\end{array}$ & - & EPC12 & 1 \\
\hline $\begin{array}{c}\text { Teste baseado no triângulo de } \\
\text { Pascal [Barry 2006] }\end{array}$ & - & EPC06 & 1 \\
\hline Doutor Scratch & - & $\mathrm{EPC} 07$ & 1 \\
\hline $\begin{array}{l}\text { Avaliação de Conhecimento } \\
\text { [Chipman et al. 2019] }\end{array}$ & - & $\mathrm{EPC} 25$ & 1 \\
\hline $\begin{array}{l}\text { Computer Science Attitude } \\
\text { Survey [Wiebe et al. 2003] }\end{array}$ & - & $\mathrm{EPC} 25$ & 1 \\
\hline Solve-Its instrument [Bers. 2012] & - & EPC08 & 1 \\
\hline $\begin{array}{l}\text { Test Visual Blocks and Robotics } \\
\text { (TVBR) }\end{array}$ & - & EPC10 & 1 \\
\hline $\begin{array}{l}\text { Computational Thinking Test } \\
\text { [Román-Gonzalez et al. 2017] }\end{array}$ & - & EPC11 & 1 \\
\hline $\begin{array}{l}\text { Conceptual Foundations of Coding } \\
\text { (CFC) }\end{array}$ & - & EPC16 & 1 \\
\hline
\end{tabular}

\footnotetext{
${ }^{15}$ Bebras é uma iniciativa internacional que visa promover a Informática (Ciência da Computação, ou Computação) e o pensamento computacional entre alunos de todas as idades. Os participantes geralmente são supervisionados por professores que podem integrar o desafio Bebras em suas atividades de ensino. $\mathrm{O}$ desafio é realizado em escolas por meio de computadores ou dispositivos móveis.
} 
Os testes são utilizados para medir o desenvolvimento do PC, buscando um valor quantitativo para o aumento do PC do indivíduo, onde é aplicado um questionário e depois uma medição/técnica estatística, na qual verifica se os dados da amostra fornecem evidência suficiente para que se possa aceitar como verdadeira a hipótese de pesquisa, precavendo-se, com certa segurança, de que as diferenças observadas nos dados não são meramente casuais.

QPP - Qual o panorama atual de pesquisa dos experimentos sobre o Pensamento Computacional no Ensino Básico?

Esta questão faz uma análise geral do que foi encontrado nas outras questões deste mapeamento e aprofundando sua análise.

Foram encontrados 28 estudos e a partir das questões secundárias realizadas neste MSL, é possível inferir um panorama dos experimentos feitos sobre o Pensamento Computacional no Ensino Básico, respondendo assim a QPP deste artigo. Essas questões foram pensadas, com foco no Desafio apontado pela SBC de levar acesso participativo e universal de todo cidadão brasileiro ao conhecimento [SBC 2015, p.17].

Em relação aos testes para avaliar o $\mathrm{PC}$, o próprio número de estudos selecionados (28) revela que ainda é necessário um maior amadurecimento nos experimentos com PC para que se meça o desenvolvimento do PC por instrumentos quantitativos e também validados, já que dos 28 estudos relevantes, em 7 (1 estudo brasileiro e 6 estudos estrangeiros) foram utilizados testes feitos pelos próprios autores, o que pode comprometer a criação de evidências do desenvolvimento do PC. Além disso, é necessário uma maior padronização nos testes sobre PC, já que cada um dos testes podem medir PC em dimensões diferentes, pois podem partir de diferentes definições de componentes que formam o PC. Logo, medir o desenvolvimento do PC é importante para traçar metas palpáveis e ajudar no cumprimento do Desafio proposto pela SBC.

O material mais utilizado nos artigos relevantes deste MSL são as atividades desplugadas, levando em consideração o contexto geral (estudos estrangeiros e brasileiros) com 9 artigos, logo tal resultado pode ser pelo fato que atividades desplugadas utilizam materiais de fácil acesso em ambiente escolar (tesoura, papel, etc.), assim possibilitando a replicação dessas práticas em qualquer ambiente escolar e facilitando o uso pelos professores e dos alunos [Rodrigues et al. 2018], os quais podem ter dificuldades no uso de materiais digitais devido a sua própria falta de inclusão digital ou possíveis diferenças sócio-econômicas, além do custo ser possivelmente menor para a realização do experimento ao usar materiais do cotidiano escolar [de Souza and Nunes 2019]. Em contexto estrangeiro, a preferência é o uso de materiais digitais, principalmente do Scratch e de robôs/kit de robótica, denotando um maior investimento na pesquisa e uma possível cultura digital maior que no contexto brasileiro. A falta de investimento em pesquisa dificulta a realização do Desafio proposto, já que para levar acesso ao conhecimento a todo cidadão brasileiro "existem barreiras tecnológicas, educacionais, culturais, sociais e econômicas, que impedem o acesso e a interação"[SBC 2015, p.17].

Em relação às disciplinas nas quais se aplicam os experimentos, é necessário buscar uma maior interdisciplinaridade tanto em contexto brasileiro quanto estrangeiro, relacionando o ensino de computação às matérias curriculares, fomentando as competências relatadas pela BNCC e buscando atividades que possam se aproximar da realidade do 
público-alvo dos experimentos. Além disso, é necessário mais experimentos fora do eixo STEM, buscando assim o desenvolvimento de outras habilidades como as habilidades socioemocionais, as quais também podem ser importantes para o mercado de trabalho, já que o Desafio proposto propõe acesso participativo e universal ao conhecimento, é necessário também que o cidadão consiga por meio desse conhecimento dialogar e viver em sociedade.

\section{Ameaças a Validade}

No desenvolvimento deste artigo, limitações podem ter afetado os resultados obtidos: (1) Seleção dos estudos relevantes: Foram selecionados estudos através de critérios de inclusão e exclusão e uma checagem por mais de um pesquisador oferecido pelo SUMARIZE. Além disso, houve a tentativa na utilização de curingas $(*$, ?) para a busca de palavraschave no plural, porém algumas bases não os aceitaram; (2) Externa: O mapeamento foi realizado com limitação temporal, foram catalogados estudos do ano de 2019 até maio de 2021, para assim traçar um panorama com pesquisas mais recentes; e (3) Extração dos dados: Foi realizada pelas ferramentas de extração no SUMARIZE e foram adicionadas as informações relevantes ligadas às questões de pesquisa.

\section{Conclusão}

O PC está presente na BNCC como uma habilidade que deve ser estimulada nos alunos, de forma integrada a outras disciplinas e como forma de promover o cumprimento do Desafio proposto pela SBC. Neste contexto, este artigo teve como objetivo realizar um Mapeamento Sistemático da Literatura (MSL) de artigos do estado da arte sobre experimentos com Pensamento Computacional no Ensino Básico.

Por meio dos estudos relevantes, foi observado informações importantes para os experimentos sobre PC: há uma falta de interdisciplinaridade, pois a maioria dos experimentos não está integrada a nenhuma disciplina, porém a maioria das tarefas propostas dentro do experimentos estão no eixo STEM, tanto em contexto brasileiro quanto estrangeiro, apesar de haverem experimentos na área de Música, Dança e Português; atividades desplugadas são utilizadas na maior parte dos experimentos, logo seguido pela linguagem de programação Scratch; há uma diversidade de testes usados para medir o Pensamento Computacional antes e após os experimentos, porém em sua maioria são testes criados pelos próprios autores e sem nenhuma validação.

Como possibilidade de estudos futuros, é necessário trazer mais interdisciplinaridade para os experimentos e que possam ser realizados fora do eixo STEM, tanto em contexto brasileiro quanto estrangeiro. É também necessário em contexto brasileiro mais experimentos com materiais que sejam diferentes de atividades desplugadas e que também possam utilizar materiais digitais, o que leva há um maior investimento em pesquisa e ao contato com tecnologias que podem ser desconhecidas para os participantes. Além disso, se faz presente a necessidade de discussão de quais componentes o PC trabalha para que assim haja uma formalização do que um teste avaliando PC deve medir, sendo que os testes encontrados mediam PC com perspectivas diferentes.

\section{Referências}

BA, K. and Charters, S. (2007). Guidelines for performing systematic literature reviews in software engineering. 2. 
Barry, P. (2006). On integer-sequence-based constructions of generalized pascal triangles, j. integer sequences. Journal of integer sequences, 9.

Bers, M. U. (2012). Designing digital experiences for positive youth development: From playpen to playground. Oxford University Press.

Brackmann, C. P. (2017). Desenvolvimento do pensamento computacional através de atividades desplugadas na educação básica.

Brasil (2017). Infraestrutura: Equipamentos, internet e condições gerais.

CEIE (2018). Comissão especial de informática na educação.

Chipman, H. E., Rodríguez, F. J., and Boyer, K. E. (2019). "i impressed myself with how confident i felt": Reflections on a computer science assessment for k-8 teachers. In Proceedings of the 50th ACM Technical Symposium on Computer Science Education, SIGCSE '19, page 1081-1087, New York, NY, USA. Association for Computing Machinery.

CIEB (2016). Centro de inovação para a educação brasileira.

Cuny, J., Snyder, L., and Wing, J. M. (2010). Demystifying computational thinking for non-computer scientists. Unpublished manuscript in progress, referenced in http://www. cs. cmu. edu/ CompThink/resources/TheLinkWing. pdf.

de França, R. and Tedesco, P. (2015). Explorando o pensamento computacional no ensino médio: do design à avaliação de jogos digitais. In Anais do XXIII Workshop sobre Educação em Computação, pages 61-70. SBC.

de Freitas, K. A., de Almeida Vilela, B., Ladeira, M. B., and de Araújo Carvalho, T. (2017). Gerenciamento de processo de negócio e seu impacto no desempenho organizacional: Uma revisão da literatura. ANAIS SIMPAC, 7(1).

de Oliveira, M., de Souza, A., Ferreira, A., and Barreiros, E. (2014). Ensino de lógica de programação no ensino fundamental utilizando o scratch: um relato de experiência. In Anais do XXII Workshop sobre Educação em Computação, pages 239-248. SBC.

de Souza, F. F. and Nunes, M. A. S. N. (2019). Práticas e resultados obtidos na aplicação do pensamento computacional desplugado no ensino básico: Um mapeamento sistemático. In Brazilian Symposium on Computers in Education (Simpósio Brasileiro de Informática na Educação-SBIE), volume 30, page 289.

França, R. and Tedesco, P. (2017). Pensamento computacional sob a perspectiva de licenciandos em computação. In Anais do Workshop de Informática na Escola, volume 23, page 795 .

Kale, U. and Yuan, J. (2021). Still a new kid on the block? computational thinking as problem solving in code.org. Journal of Educational Computing Research, 59(4):620644. cited By 1.

Korkmaz, Ö., Çakir, R., and Özden, M. Y. (2017). A validity and reliability study of the computational thinking scales (cts). Computers in human behavior, 72:558-569.

MEC (2019). Base nacional comum. 
Mecca, G., Santoro, D., Sileno, N., and Veltri, E. (2021). Diogene-ct: tools and methodologies for teaching and learning coding. International Journal of Educational Technology in Higher Education, 18(1). cited By 0.

OECD (2020). What Students Learn Matters.

Papert, S., Solomon, C., Soloway, E., and Spohrer, J. (1971). Twenty things to do with a computer. Cambridge, MA.

Petersen, K., Feldt, R., Mujtaba, S., and Mattsson, M. (2008). Systematic mapping studies in software engineering. In 12th International Conference on Evaluation and Assessment in Software Engineering (EASE) 12, pages 1-10.

Raabe, A., Santana, A. L. M., Ellery, N., and Gonçalves, F. (2017). Um instrumento para diagnóstico do pensamento computacional. In Anais dos Workshops do Congresso Brasileiro de Informática na Educação, volume 6, page 1172.

Relkin, E., de Ruiter, L., and Bers, M. (2021). Learning to code and the acquisition of computational thinking by young children. Computers and Education, 169. cited By 0 .

Rich, P., Mason, S., and O'Leary, J. (2021). Measuring the effect of continuous professional development on elementary teachers' self-efficacy to teach coding and computational thinking. Computers and Education, 168. cited By 1.

Rodrigues, S., Aranha, E., and Silva, T. R. (2018). Computaçao desplugada no ensino de programaçao: Uma revisao sistemática da literatura. In Brazilian Symposium on Computers in Education (Simpósio Brasileiro de Informática na Educação-SBIE), volume 29, page 417.

Román-González, M., Pérez-González, J.-C., and Jiménez-Fernández, C. (2017). Which cognitive abilities underlie computational thinking? criterion validity of the computational thinking test. Computers in human behavior, 72:678-691.

Romero, M. (2016). De l'apprentissage procédural de la programmation à l'intégration interdisciplinaire de la programmation créative. Formation et profession: revue scientifique internationale en éducation, 24(1):87-89.

Romero, M., Vallerand, V., and Nunes, M. (2019). Almanaque para popularização de ciência da computação série 12: Guia pedagoǿgico e tecnológica; volume 1: Atividades tecnocriativas para crianças do século 21. ed. 1.

Rowe, E., Almeda, M., Asbell-Clarke, J., Scruggs, R., Baker, R., Bardar, E., and Gasca, S. (2021). Assessing implicit computational thinking in zoombinis puzzle gameplay. Computers in Human Behavior, 120. cited By 1.

SBC (2015). Grandes desafios da pesquisa em computação no brasil - 2006 - 2016.

SBC (2018). Sociedade brasileira de computação.

Serin, O., Serin, N. B., and Sayg1l1, G. (2010). Developing problem solving inventory for children at the level of primary education (psic). Elementary Education Online, 9(2).

Silva, I. et al. (2018). Almanaque para popularização de ciência da computação. série 6: Metodologia científica e tecnológica; volume 7: Mapeamento sistemático - parte 1. 
Wiebe, E., Williams, L. A., Yang, K., and Miller, C. S. (2003). Computer science attitude survey. Technical report, North Carolina State University. Dept. of Computer Science.

Wing, J. M. (2006). Computational thinking. Commun. ACM, 49(3):33-35.

Yağc1, M. (2019). A valid and reliable tool for examining computational thinking skills. Education and Information Technologies, 24(1):929-951. 